\title{
Das DSM-5: Was ist neu?
}

Das Diagnostische und Statistische Manual für Psychische Störungen ist ein von der American Psychological Association veröffentlichtes Klassifikationssystem zur Diagnose psychischer Störungen. 2013 wurde die 5. Auflage publiziert. Von der Aktualisierung der bisherigen sowie der Einführung mehrerer neuer Kategorien psychischer Störungen sind nicht nur Psychologen und Psychiater betroffen, sondern deutlich mehr als bisher auch Allgemeinmediziner, Pharmakologen sowie das Sozial-, Schul- und Gerichtswesen.

\section{Jana Bryjova ${ }^{a}$, \\ Simone Munsch ${ }^{b}$ \\ a Klinische Psychologin \\ b Prof. Dr. Ordinaria für Klinische Psychologie und Psychotherapie}

\footnotetext{
* Die Literatur findet sich unter www.saez.ch $\rightarrow$ Aktuelle Nummer oder $\rightarrow$ Archiv $\rightarrow 2014 \rightarrow 15$.
}

Korrespondenz:

Dipl.-Psych. Jana Bryjova Universität Freiburg Departement für Psychologie Lehrstuhl für Klinische Psychologie und Psychotherapie 2 , rue de Faucigny CH-1700 Freiburg Tel. 0263007661

jana.bryjova[at]unifr.ch

\section{Das DSM-5}

Das Diagnostische und Statistische Manual für Psychische Störungen (DSM) stellt ein für die klinisch psychologische/psychiatrische Diagnostik und den entsprechenden Forschungsbereich massgebliches Klassifikationssystem psychischer Störungen dar. Die einzelnen Kategorien wurden seit 1952 in regelmässigen Abständen überarbeitet und aktualisiert. Um den seit mehr als 30 Jahren bestehenden Wissenszuwachs in der Ätiologie, Epidemiologie und Therapie der wichtigsten psychischen Störungen zu adaptieren, begann im Jahre 2011 eine Revidierung der seit 2000 bestehenden vierten Version des DSMIV-TR [1]*. Mehr als 160 Forschungsexperten und Kliniker aus verschiedenen akademischen und medizinischen Institutionen (darunter z. B. Neuropsychologie, Genetik, Biologie, Statistik, Epidemiologie und Sozialwissenschaften) wurden eingeladen, sich zu beteiligen. Noch nie in der Entwicklungsgeschichte eines diagnostischen Kodiersystems haben sich so viele spezialisierte Forscher und Kliniker in entsprechend umfangreichem zeitlichem und inhaltlichem Rahmen mit der Verbesserung der diagnostischen Standards beschäftigt. Die Priorität der Erstellung dieses Manuals lag von Anfang an in der Optimierung der praktischen Anwendbarkeit, wobei gleichzeitig die Kontinuität der diagnostischen Tradition bewahrt werden sollte. Überdies wurden die Diagnosen an die neuen Forschungsergebnisse adaptiert und solche, die bisher als «nicht anders spezifiziert» galten, wurden revidiert, um ihre Reliabilität zu verbessern. Nichtsdestotrotz hat das neueingeführte System bereits viel Kritik geerntet.

\section{Labeling oder bessere Behandlungsaussichten?} Die grundsätzlichen Änderungen, die in der fünften Fassung des Klassifikationssystems vorgenommen worden sind, werden Konsequenzen mit sich bringen, die nicht nur für Fachpersonen relevant sind. In der breiten Öffentlichkeit (z.B. im Spiegel oder The New York Times) ist bereits scharfe Kritik an dem neuen Manual geäussert worden. Insbesondere wird hingewiesen auf die Gefahr einer Pathologisierung der Gesellschaft durch die Einführung neuer Störungskategorien und der Senkung der Schwelle, die zur Diagnose einer psychischen Störung notwendig ist (z. B. Verkürzung der Anzahl bzw. der Dauer der Symptome). Laien, aber auch viele Fachpersonen befürchten, dass zukünftig viele Menschen mit einer psychischen Diagnose «abgestempelt» werden. Viele Experten erwarten daher einen Anstieg der Prävalenzraten der häufigsten psychischen Störungen.

Zu den bekanntesten Kritikern des DSM-5 gehört A. Frances, ein US-amerikanischer Psychiater, der selber die vierte Revision des DSM durchgeführt hatte. Frances bezeichnet den Publikationszeitpunkt des DSM-5 als «(...) a sad moment for psychiatry and a risky one for patients» [2]. Frances warnt vor Inflation der psychischen Störungen durch die Einführung neuer Störungsbilder und der Senkung der bisherigen Schwellen und rät den Klinikern davon ab, das Manual in seiner fünften Fassung zu benutzen.

Einige Psychiater, Psychologen und Psychoanalytiker in Frankreich haben eine Gruppe «Stop DSM» gebildet und initiierten im Internet eine Petition gegen die Einführung des DSM-5 (www.stop-dsm.org). Weitere Opponenten sind feministische Autorinnen, die besonders im historischen Kontext des Feminismus ihre Aufgabe darin sehen, auf den Einfluss der dominanten gesellschaftspolitischen Ideologien der jeweiligen Zeit auf die Regulierung des problematischen (d.h. als nicht «normal» eingestuften) Verhaltens hinzuweisen [3]. Laut Jane Ussher [4], Professorin an der University of Western Sydney, besteht bei Anlehnung an DSM-5 bei Frauen eine signifikant höhere Wahrscheinlichkeit (im Vergleich zu Männern), die Diagnose einer Depression, Angststörung, Essstörung, Borderline-Persönlichkeitsstörung, Somatoformen Störung oder Schlafstörung zu erhalten und somit ein «labeling» zu erfahren. Frau Ussher verdeutlicht in diesem Zusammenhang die Gefahren der voreiligen Medikation und deren Nebeneffekte. 
Trotz der anfänglichen Skepsis wird von vielen klinisch und wissenschaftlich tätigen Psychologen und Psychiatern die Aktualisierung allerdings begrüsst, so sollen die relaxierten Kriterien und die damit verbundene vermehrte Klassifizierung von psychischen Störungen die Entwicklung und Überprüfung neuer Therapieprogramme fördern. Dies gilt insbesondere für Kurztherapien und Präventionsprogramme, deren Relevanz durch die Erfassung leichterer Ausprägungen psychischer Störungen im DSM-5 mehr Beachtung erhalten.

Dr. Sederer zählt zu den Befürwortern des neuen Kodiersystems. Er ist Medizinischer Direktor des New York State Office of Mental Health (OMH). Professor an der Columbia/Mailman Public School of Health, Autor von mehreren Büchern für Fachleute und Laien und ein Mitglied der APA. In seinen Blogs unterstreicht Sederer [5] die Notwendigkeit einer einheitlichen Sprache für die Vergabe der psychiatrischen Diagnosen, um valide und reliable Diagnosen und damit die bestmögliche Behandlung zu gewährleisten. Die Diagnosevergabe einer psychischen Störung muss gemäss Sederer [5] nicht zwangsläufig zu einer Stigmatisierung führen. Sie kann genauso gut einen sozial akzeptierten Grund für verminderte Leistungs- oder Funktionsfähigkeit eines Individuums liefern: Ähnlich wie nach einem Myokardinfarkt kann durch die Diagnosevergabe der Major Depression die Notwendigkeit der Hospitalisierung verdeutlicht werden.

\section{Die wichtigsten Veränderungen}

Trotz breiter Verwendung des multiaxialen Vorgehens bei der Vergabe der psychischen Diagnosen im DSM-IV weicht das DSM-5 von dieser Tradition bewusst ab, mit der Begründung, dass es fundamentale Unterschiede in der Konzeptualisierung der einzelnen Achsen gab und die psychischen Störungen ohne Bezug auf die zugrundeliegenden biologischen oder physischen Faktoren dargestellt waren. Die neue Herangehensweise sei ausserdem konsistent mit den bereits etablierten Richtlinien der WHO und ICD und erfasst den individuellen Zustand bzw. die Funktionsfähigkeit separat von der erteilten Diagnose [6]. Überarbeitet und aktualisiert wurden folgende psychische Störungen: Depressive Störungen, Essstörungen, Somatoforme Störungen, SchlafWach-Störungen, Sucht- und zugehörige Störungen.

Interaktiver

Wollen Sie diesen Artike kommentieren? Nutzen Sie dafür die Kommentarfunktion in der Online Version oder sehen Sie nach, was Ihre Kolleginnen und Kollegen bereits geschrieben haben: www.saez.ch/ aktuelle-ausgabe/ interaktive-beitraege/ dass eine frühe und präzise Diagnostik neben der Stimulation von Forschungsprojekten auch mit einem positiveren Verlauf assoziiert sein kann. Gilt doch die Chronizität bei der Behandlung der meisten psychischen Störungen als ein negativer Prädiktor. Die Behandlungskosten einer chronifizierten psychischen Störung sind in den meisten Fällen höher und die ökonomischen, sozialen und psychischen Folgen viel schwerwiegender und weitreichender [7].

Dem DSM-5 stand noch eine letzte Prüfung im Oktober 2013 bevor. An diesem Datum wurde das DSM mit der revidierten Version des ICD-10 [8] verlinkt.

\section{Zusammenfassung und Ausblick}

Ohne ein diagnostisches Manual zur Klassifikation und Kategorisierung psychischer Störungen wird heutzutage kein Kliniker mehr auskommen. Nicht nur Forscher, sondern auch die behandelnden Experten bemühen sich um eine Einordnung der beobachteten Symptomatik, um dem Patienten Zugang zu Behandlung und Unterstützung zu gewährleisten, um sich mit anderen Experten über eine angemessene Behandlung und Prognose austauschen zu können und schliesslich, um die festgestellte Diagnose dem Patienten (und seiner Familie) in adäquater Form mitzuteilen. Den Sinn und Zweck der fünften Version des DSM fasst der Präsident der American Psychiatric Association A. Schatzberg [9] folgendermassen zusammen: «(...) Our focus is not on keeping things as they are but instead on determining what is working with the current DSM, what is not working, and (...) how to correct what is not working.»

In vielen Fällen hat das bisherige kategoriale Diagnosesystem nur ansatzweise die klinische Realität widergespiegelt. Zum Beispiel leiden viele Patienten mit einer Schizophrenie gleichzeitig an einer Schlafstörung, oder Patienten mit einer Major Depression weisen in vielen Fällen eine signifikante Angstsymptomatik auf. Die Einführung der dimensionalen Kriterien für psychische Störungen im DSM-5 entspricht demnach in einem grösseren Ausmass der Realität und legt einen besonderen Fokus auf die Prävention der Störungen. Dadurch wird die Relevanz der Früherkennung betont und entsprechende Fachpersonen werden angesprochen, wie z. B. Allgemeinmediziner und Hausärzte, die häufig die erste Anlaufstelle für Patienten mit psychischen Problemen darstellen [10]. Eine spezifische Schulung dieser Berufsgruppe zur Früherkennung und zur Durchführung erster Interventionen könnte künftig eine zentrale Rolle einnehmen.

Möchten Sie Ihre diagnostischen Fähigkeiten im Bereich der häufig vorkommenden psychischen Störungen überprüfen? Nehmen Sie an einer Studie der Universität Fribourg für Hausärzte teil: Sehen Sie sich ein Video an, in dem der Patient/die Patientin über seine/ihre Beschwerden berichtet und versuchen Sie, anhand der geschilderten Symptomatik eine Diagnose zu stellen: www.unifr.ch/psychotherapie/ hausarztstudie 


\section{Literatur}

1 Saß H, Wittchen HU, Zaudig M, Houben I. DSM-IV-TR. Diagnostische Kriterien. Göttingen: Hogrefe; 2003.

2 Frances A. Opening Pandora's box: The 19 worst suggestions for DSM-5. Psychiatric Times. 2013; 27.

3 Marecek J. Special Issue: DSM-5 and Beyond: A Critical Feminist Engagement with Psychodiagnosis Guest Editors: Jeanne Marecek and Nicola Gavey Editorial Introduction; 2013.

4 Ussher J. DSM-5 helps perpetuate the myth of women's madness. The Conversation; 2013.

5 Sederer LI. The American Psychiatric Association's New Bible; 2011.
6 APA. DSM 5: American Psychiatric Association; 2013.

7 Margraf J. Kosten und Nutzen der Psychotherapie. 2009.

8 WHO. International statistical classification of diseases and related health problems: World Health Organization; 2004.

9 Schatzberg AF, Scully JH, Kupfer DJ, Regier DA. Setting the Record Straight: A Response to Frances Commentary on DSM-V. Psychiatric Times; 2011.

10 Kupfer DJ, Regier DA. (2010) Why all of medicine should care about DSM-5. JAMA. 2010;303:1974-5. 